\title{
BLICKDIAGNOSE
}

\author{
Exophthalmus, Bauch-, Knochen- \\ und Kopfschmerzen
}

\section{Welche Organe sind hier betroffen?}

\section{Ein 50-jähriger Patienten wird wegen Kno- chenschmerzen beider Oberschenkel und Kniegelenke, Kopfschmerzen, abdominellen Schmerzen und einem bilateralen Exophthal- mus stationär aufgenommen.}

— Die Röntgenaufnahme beider Kniegelenke mit proximalen Oberschenkeln beidseits zeigte eine bilaterale symmetrische Sklerose der Femurdiaphysen (Abb. 1). Aufgrund der abdominellen Schmerzen wurde eine Computertomografie des Thorax, Abdomens und Beckens durchgeführt, welche eine ausgedehnte Raumforderung des Mediastinums und des Retroperitoneums aufwies. Die anschließende Punktion der retroperitonealen Raumforderung konnte histologisch eine Erdheim-ChesterErkrankung bestätigen.

Die Lipogranulomatosis als Erdheim-Chester-Erkrankung ist eine seltene Form der Langerzellhistiozytose, welche mit einer Proliferation von cholesterinhaltigen Histiozyten verbunden ist. Die Ätiologie ist bis heute ungeklärt. Diese Erkrankung betrifft vor allem Patienten ab dem 40. Lebensjahr. Die klinischen Symptome weisen ein breites Spektrum, von asymptomatischen Patienten bis zu einer Multiorganmanifestation mit entsprechender Funktionseinschränkung der betroffenen Organe, auf.

Charakteristisch für die Erdheim-Chester-Erkrankung sind Knochenschmerzen, welche durch eine milde, aber permanente Schmerzsymptomatik in der Knieregion gekennzeichnet sind. Röntgenologisch fassbare Stigmata sind eine bilateral symmetrische Sklerose der langen Röhrenknochen der unteren Extremität unter Aussparung des Achsenskelettes. In einigen Fällen weisen diese Patienten auch einen Befall des Hand- und Fußskelettes auf.

Neben der ossären Manifestation sollte auch eine extraossäre Beteiligung der Erdheim-ChesterErkrankung bei allen Patienten evaluiert werden.

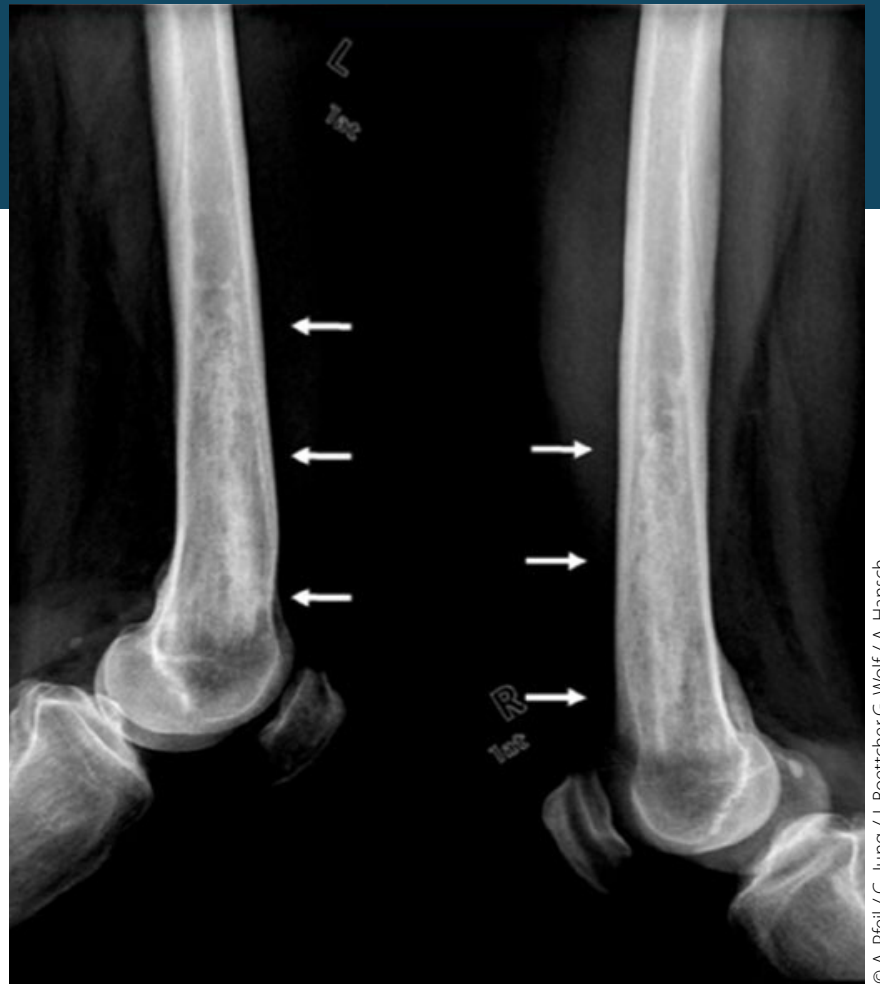

Abb. 1 Röntgenbilder der Knie und der Femora beidseits mit Nachweis einer bilateralen symmetrischen Sklerose (Pfeile).

Hierbei sollten folgende Organsysteme untersucht werden: (I) Hypothalamische-hypophysäre Achse, (II) Lunge (Ausschluss Lungenfibrose), (III) Herz (Ausschluss einer myokardialen oder perikardialen Beteiligung), (IV) Leber, (V) Niere, (VI) Milz, (VII) Retroperitoneum (Ausschluss einer retroperitonealen Fibrose) und (VIII) Auge (Ausschluss eines ophthalmologischen Befalls).

Keywords: Chester-Erdheim disease

- Alexander Pfeil ${ }^{1}$, Christian Jung ${ }^{2}$, Joachim Boettcher ${ }^{3}$, Gunter Wolf ${ }^{1}$, Andreas Hansch ${ }^{4}$

${ }^{1}$ Klinik für Innere Medizin III, Universitätsklinikum Jena

${ }^{2}$ Klinik für Innere Medizin I, Universitätsklinikum Jena

${ }^{3}$ Institut für Diagnostische und Interventionelle Radiologie, SRH WaldKlinikum Gera

${ }^{4}$ Institut für Diagnostische und Interventionelle Radiologie, Universitätsklinikum Jena

www.springermedizin.de

Weitere Blickdiagnosen finden Sie im

Internet unter:

http://www.springermedizin.de/blickdiagnose 